\title{
Atypical Composition and Ultrastructure of Proteoglycans in the Mouse Corneal Stroma
}

\author{
Robert D. Young, ${ }^{1}$ Debbie Tudor, ${ }^{2}$ Antbony J. Hayes, ${ }^{2}$ Briedgeen Kerr, ${ }^{2}$ \\ Yasutaka Hayashida, ${ }^{3}$ Kohji Nishida, ${ }^{3}$ Keith M. Meek, ${ }^{1}$ Bruce Caterson, ${ }^{2}$ \\ and Andrew J. Quantock ${ }^{1}$
}

Purpose. Recently, gene-targeted strains of mice with null mutations for specific proteoglycans (PGs) have been used for investigations of the functional role of these molecules. In the present study, the corneal stroma of the mouse was examined to provide some baseline PG morphologies in this species.

Methods. Monoclonal antibodies to specific glycosaminoglycan (GAG) chain sulfation patterns were used to characterize PG composition in corneal extracts by SDS-PAGE and Western blot analysis and to identify their tissue distribution by immunofluorescence microscopy. PGs were also visualized by transmission electron microscopy after contrast enhancement with cationic dye fixation.

Results. Western blot analysis of pooled corneal extracts and immunofluorescence of tissue sections identified 4-sulfated, but not 6-sulfated, chondroitin sulfate/dermatan sulfate (CS/ DS). Keratan sulfate (KS) was present only as a low-sulfated moiety. Electron microscopic histochemistry disclosed a complex array of corneal PGs present as (1) fine filaments radiating from collagen fibrils, and (2) elongate, straplike structures, running either along the fibril axis or weaving across the primary fibril orientation. These large structures were digested by chondroitinase $\mathrm{ABC}$, but not by keratanase.

Conclusions. $\mathrm{KS}$ in the mouse is predominantly undersulfated and generates an immunostaining pattern that differs from that observed in corneas of other mammalian species thus far investigated. The mouse cornea resembles other mammalian corneas in the presence of filamentous arrays of small, collagen-associated stromal PGs visualized by cationic dye staining. However, large dye-positive structures with a CS/DS component are also present and appear to be unique to the cornea of this species. (Invest Ophthalmol Vis Sci. 2005;46:1973-1978) DOI:10.1167/iovs.04-1309

From the ${ }^{1}$ School of Optometry and Vision Sciences, and ${ }^{2}$ School of Biosciences, Cardiff University, Cardiff, United Kingdom; and the ${ }^{3}$ Department of Ophthalmology, Osaka University Medical School, Osaka, Japan. All Cardiff-based authors are members of the Cardiff Institute of Tissue Engineering and Repair, Cardiff University, Cardiff, United Kingdom.

Supported by the Biotechnology and Biological Sciences Research Council Grant 72/B18021 (AJQ, BC).

Presented at the annual meeting of the Association for Research in Vision and Ophthalmology, Fort Lauderdale, Florida, April 2004.

Submitted for publication November 9, 2004; revised January 20, 2005; accepted January 20, 2005.

Disclosure: R.D. Young, None; D. Tudor, None; A.J. Hayes, None; B. Kerr, None; Y. Hayashida, None; K. Nishida, None; K.M. Meek, None; B. Caterson, None; A.J. Quantock, None

The publication costs of this article were defrayed in part by page charge payment. This article must therefore be marked "advertisement" in accordance with 18 U.S.C. $\$ 1734$ solely to indicate this fact.

Corresponding author: Andrew J. Quantock, Structural Biophysics Group, School of Optometry and Vision Sciences, Cardiff University, Redwood Building, King Edward VII Ave., Cardiff CF10 3NB, UK; quantockaj@cardiff.ac.uk.
$\mathrm{P}$ roteoglycans (PGs) are key components of the corneal stroma where, as in other connective tissues, they contribute to the interfibrillar compartment of the matrix. The internal fine structure of the corneal stroma has functional significance, with the uniform diameter and characteristic spatial organization of collagen fibrils believed to be essential for tissue transparency. ${ }^{1-4}$ Much evidence points to a role for PGs in the regulation of tissue architecture through interactions with fibrillar collagen and control of fibril $\operatorname{size}^{5-7}$ and arrangement. ${ }^{8-10}$

The small leucine-rich PGs in cornea are macromolecules consisting of a protein backbone to which variable numbers of repeat disaccharide - that is, glycosaminoglycan (GAG) - side chains are covalently attached. The main corneal GAGs are chondroitin sulfate/dermatan sulfate (CS/DS) and keratan sulfate (KS), the former linked to protein as decorin and biglycan, ${ }^{11,12}$ and the latter contributing to the three corneal KS PGs, lumican, ${ }^{13}$ keratocan, ${ }^{14}$ and mimecan. ${ }^{15}$ Advances in molecular genetics in recent years have allowed the generation of strains of mice with null mutations in selected PGs, and these offer many advantages for rapid progress in our understanding of the roles of PGs in the cornea. ${ }^{16-21}$ The advent of mouse strains with multiple genetic alterations will further enhance such work. However, the normal mouse cornea is thought to differ from the corneas of other, larger mammals, particularly in relation to its KS content. ${ }^{22-24}$ To underpin investigations of PG structure-function relationships using mice with experimental gene knockouts, a more comprehensive appreciation of PGs and GAGs in the corneal stroma of the normal mouse is needed. In this report we highlight some species-specific characteristics of the normal mouse corneal stroma from electron microscopy and immunofluorescence microscopy using monoclonal antibodies specific for different sulfation patterns on CS/DS- and KS-GAG chains.

\section{Methods}

Mature 12- to 15-week-old BALBc and C57/B6 strain mice were obtained from breeding colonies maintained at the School of Biosciences, Cardiff University. At all times animals were treated in accordance with the ARVO Statement for the Use of Animals in Ophthalmic and Vision Research and in compliance with local ethical regulations. Eyes were removed within 5 minutes postmortem after halothane anesthesia and cervical dislocation.

\section{Corneal Extraction and Immunoblotting}

One hundred corneas were extracted in $4 \mathrm{M}$ guanidine hydrochloride for 12 hours at $4^{\circ} \mathrm{C}$ in the presence of protease inhibitors $(0.05 \mathrm{M}$ sodium acetate, $0.01 \mathrm{M}$ disodium EDTA, $0.1 \mathrm{M}$ 6-amino hexanoic acid, $0.005 \mathrm{M}$ benzamidine $\mathrm{HCl}$, and $0.5 \mathrm{mM}$ PMSF [pH 5.8-6.8]). Supernatants were collected, and the tissue was subjected to repeat extraction for a further 12 hours at $4^{\circ} \mathrm{C}$. Pooled extracts were dialyzed into $6 \mathrm{M}$ urea in $0.1 \mathrm{M}$ Tris-HCl, 0.1\% CHAPS (3-([3-cholamidopropyl] dimethylammonio-2-hydroxy-1-propanesulfonate). The crude PG extract was applied to a diethylaminoethyl (DEAE) Sepharose fast flow anion ex- 
TABLE 1. Antibodies Used in Immunoblot Analysis and Immunohistochemistry

\begin{tabular}{|c|c|c|c|}
\hline Antibody & Epitope & Tissue Pretreatment Required & References \\
\hline $5 \mathrm{D} 4$ & $\begin{array}{l}\text { Linear pentasulfated sequences of } N \text {-acetyl } \\
\text { lactosamine disaccharides of KS PGs in which } \\
\text { both the GalNAC and Gal are sulfated }\end{array}$ & None & $25-28$ \\
\hline $1 \mathrm{~B} 4$ & $\begin{array}{l}\text { Lesser sulfated sequences of } \mathrm{N} \text {-acetyl lactosamine } \\
\text { disaccharides of KS PGs }\end{array}$ & None & $26-28$ \\
\hline 4D1 & Similar to $1 \mathrm{~B} 4$ & None & - \\
\hline $1 \mathrm{~B} 5$ & $\begin{array}{l}\text { 0-Sulfated unsaturated disaccharide "stubs" (C-0-S) } \\
\text { on CS/DS PGs }\end{array}$ & Chondroitinase $\mathrm{ABC}$ to generate neoepitope "stub" & 26 \\
\hline $2 \mathrm{~B} 6$ & $\begin{array}{l}\text { 4-Sulfated unsaturated disaccharide "stubs" (C-4-S) } \\
\text { on CS/DS PGs }\end{array}$ & Chondroitinase $\mathrm{ABC}$ to generate neoepitope "stub" & 26 \\
\hline $3 \mathrm{~B} 3$ & $\begin{array}{l}\text { 6-Sulfated unsaturated disaccharide "stubs" (C-6-S) } \\
\text { on CS/DS PGs }\end{array}$ & Chondroitinase $\mathrm{ABC}$ to generate neoepitope "stub" & 26 \\
\hline
\end{tabular}

change chromatography resin (Amersham Pharmacia Biotech, Piscataway, NJ). Bound PGs were eluted with $1.5 \mathrm{M} \mathrm{NaCl}$, then dialyzed into double-distilled water (Milli-Q; Millipore, Bedford, MA). Dimethylmethylene blue analysis revealed $60 \mu \mathrm{g}$ GAG in the preparation. The sample was freeze dried and reconstituted in $60 \mu \mathrm{L} 0.1 \mathrm{M}$ Tris acetate ( $\mathrm{pH} 6.8$ ). A portion of the sample $(30 \mu \mathrm{L})$ was digested with $0.01 \mathrm{U}$ keratanase (EC 3.2.1.103; MP Biomedicals [formerly ICN Biomedicals], Irvine, CA), and 0.01 U chondroitinase ABC (EC 4.2.2.4; Sigma-Aldrich, Poole, UK) per milligram GAG.

Digested and undigested samples were mixed with an equal volume of $2 \times$ SDS buffer ( 0.125 M Tris-HCl, $4 \%$ SDS, $20 \%$ glycerol, and $0.01 \%$ bromophenol) and $10 \% \beta$-mercaptoethanol. The samples were reduced by boiling for 5 minutes, and subsequently $3 \mu \mathrm{g}$ of GAG was loaded onto $4 \%$ to $20 \%$ Tris glycine gradient gels (Invitrogen, Renfrew, UK) for Western blot analysis using the monoclonal antibodies 5D4, 1B4, 4D1, 1B5, 2B6, and 3B3. These antibodies are well characterized $^{25-28}$ and are specific for KS or CS/DS-GAGs on PGs. They recognize discrete epitopes defined by sulfation patterns on the disaccharide chains, some of which require exposure by partial predigestion with purified enzymes (summarized in Table 1). Chondroitinase and keratanase digestion were normally used in tandem to generate the immunoepitope and facilitate antibody penetration.

Antibody $2 \mathrm{~B} 6$ can also be used to investigate the presence of both DS- and CS-GAG chains on mouse corneal CS PGs by differential digestion with chondroitinase $\mathrm{ABC}$ and $\mathrm{AC}$ II enzymes. This is possible because chondroitinase $\mathrm{ABC}$ digestion generates 2B6-reactive neoepitope from both CS- and DS-GAG chains, whereas chondroitinase AC II does not digest DS and thus only generates the neoepitope C-4-S "stub" from CS chains. Chondroitinase AC II (AC II Arthro 4.2.2.5; Seikagaku Corp., Tokyo, Japan), digestion of corneal extracts was as described earlier for chondroitinase ABC.

\section{Immunohistochemistry}

Mouse eye lenses were removed through an anterior scleral incision, and the eyes were placed in optimal cutting temperature (OCT) compound and plunge frozen in liquid nitrogen-cooled isopentane. After storage at $-20^{\circ} \mathrm{C}$, serial $8-\mu \mathrm{m}$-thick sagittal sections were prepared on a cryostat and collected on glass slides. In addition, some sections were prepared for use as positive control specimens for antibody reactivity from full-thickness slices of bovine articular cartilage, dissected from the metacarpophalangeal joints of 18-month-old steers obtained from a local abattoir.

Sections were probed with the panel of monoclonal antibodies described in Table 1 and detected with FITC-conjugated goat antimouse Fab-FITC (Dako, High Wycombe, UK). Background signal, owing to labeling of endogenous mouse IgG by anti-mouse secondary antibody was avoided, by using the method of Hierck et al. ${ }^{29}$ Briefly, antibody supernatants and ascites fluids were diluted 1:10 and 1:500, respectively, with PBS and the secondary FITC-conjugated goat antimouse antibody was diluted 1:50 in this solution. Primary and secondary antibodies were complexed overnight at $4^{\circ} \mathrm{C}$. Heat-inactivated normal mouse serum was added to $0.1 \%$ concentration and mixed at room temperature for 30 minutes to absorb uncomplexed secondary antibody. Thawed, unfixed sections, with or without predigestion for 2 to 3 hours at $37^{\circ} \mathrm{C}$ with $0.4 \mathrm{U} / \mathrm{mL}$ chondroitinase $\mathrm{ABC}$ or chondroitinase AC II $(0.4 \mathrm{U} / \mathrm{mL})$, or keratanase $(0.04 \mathrm{U} / \mathrm{mL})$, were immersed in a blocking buffer for 30 minutes at room temperature $(150 \mathrm{mM} \mathrm{NaCl}, 10$ $\mathrm{mM}$ Tris-HCl, $5 \mathrm{mM}$ EDTA, and $0.05 \%$ [ $\mathrm{vol} / \mathrm{vol}]$ Tween-20 [pH 8.0]). After sections were washed in PBS, they were incubated in the antibody mixture for a minimum of 4 hours at room temperature in a humid chamber. Nuclei in sections were counterstained with Hoechst 33258 before mounting in antifade medium.

\section{Electron Microscopy}

For visualization of PGs, corneas were isolated and fixed for approximately 18 hours in $2.5 \%$ glutaraldehyde in $25 \mathrm{mM}$ sodium acetate buffer ( $\mathrm{pH} 5.7$ ), with $0.1 \mathrm{M}$ magnesium chloride and $0.05 \%$ cupromeronic blue. ${ }^{2-24}$ After the specimens were washed in buffer, contrast enhancement of stained PG-dye complexes was performed by 15-minute washes of fixed tissue in aqueous, then 50\% ethanolic $0.5 \%$ sodium tungstate solutions. Specimens were dehydrated in an ascending ethanol series and, through propylene oxide, embedded in Araldite resin. To investigate the PG composition of stained complexes, some samples were first exposed to predigestion with GAG-degrading enzymes to remove specific PG components of the cupromeronic blue staining pattern. To facilitate enzyme penetration, this procedure was performed on 50- $\mu \mathrm{m}$ cryostat sections taken through the center of the cornea, collected on glass slides, and digested for 4 hours at $37^{\circ} \mathrm{C}$ with either keratanase $(1 \mathrm{U} / \mathrm{mL}$ in Tris-acetate buffer $[\mathrm{pH} 8.0])$ to degrade $\mathrm{KS}$, or chondroitinase $\mathrm{ABC}(2.5 \mathrm{U} / \mathrm{mL}$ in Tris-acetate buffer $[\mathrm{pH} 7.4])$, to degrade 0 -, 4- and 6-sulfated CS and DS. Corneal sections were then fixed and stained as described earlier and detached from slides for resin embedding. Ultrathin sections ( $~ 90 \mathrm{~nm}$ thick) of central cornea were cut with a diamond knife, collected on copper grids, and stained with $1 \%$ PTA and $0.5 \%$ UA at $40^{\circ} \mathrm{C}$, before examination in a transmission electron microscope (EM208; Philips, Eindhoven, The Netherlands).

\section{Results}

\section{Western Blot Analysis of Mouse PG Extracts}

Mouse corneal extracts were analyzed with a range of antibodies raised against native epitopes on KS PGs that were specific for different linear sulfation motifs on the molecule. Monoclonal antibody 5D4, which is reactive with highly sulfated motifs, failed to demonstrate discrete bands, although small amounts of highly sulfated material were present, as evident by a reduction in positive immunostaining on digestion with keratanase (Fig. 1A, lanes 1, 2). In contrast, antibodies 1B4 and 4D1, which both react with lesser-sulfated KS motifs were strongly positive-again, over a broad spread of molecular mass, $\sim 70$ to 

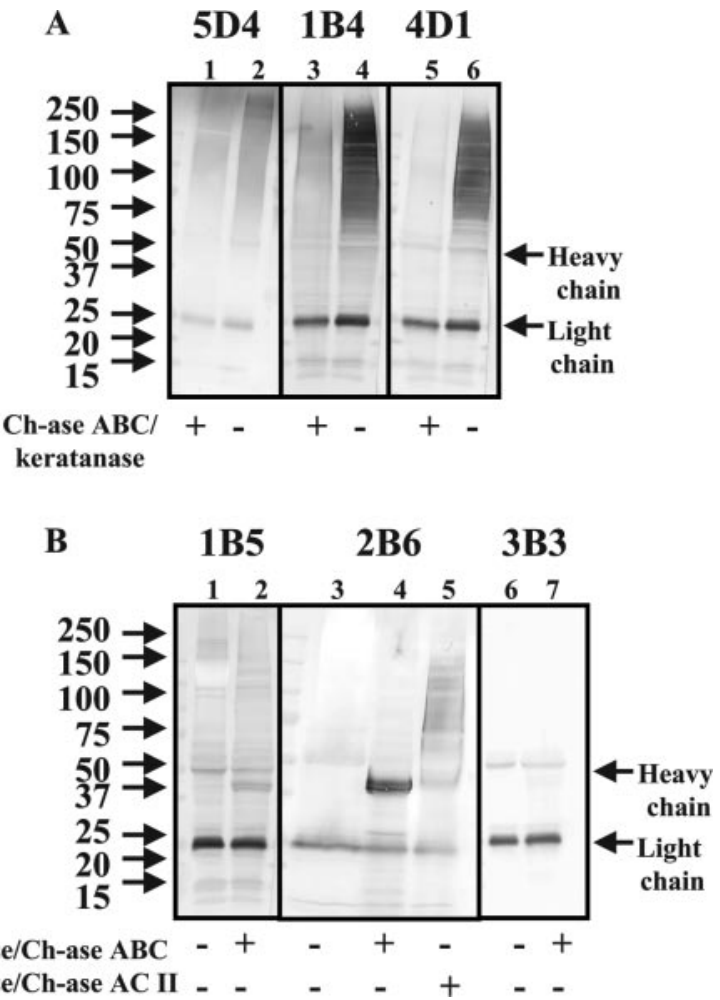

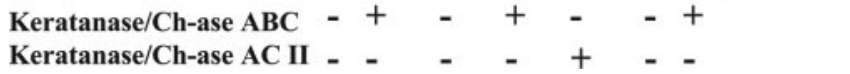

Figure 1. (A) Western blot analysis of corneal extract exposed to monoclonal antibodies specific for KS PG. Lanes 1, 3, 5: extract treated with chondroitinase and keratanase; lanes 2, 4, 6: untreated. The small amount of 5D4 reactivity indicate low levels of minimally pentasulfated disaccharides present on KS-GAG-substituted PGs. 1B4 and 4D1 showed strong reactivity for lower-sulfated KS substitution on mouse corneal KS PGs. (B) Western blot analysis of corneal extract exposed to monoclonal antibodies specific for chondroitinase-generated neoepitope stubs on chondroitin 0- (1B5), 4- (2B6) and 6-sulfate (3B3). Lanes 1, 3, 6: sample not treated with enzymes; lanes 2, 4, 7: sample digested with keratanase and chondroitinase ABC enzyme; and lane 5: sample digested with keratanase and chondroitinase AC. 4-Sulfated CS and DS contributed to a strong staining band (lane 4), which spread up the gel after chondroitinase AC digestion (lane 5) of CS, but not DS, chains. Some 0-sulfated CS stubs were present (lane 2), but CS-6-sulfate stubs were not detected on these corneal CS/DS PGs (lane 7). Bands representing IgG heavy and light chains are indicated.

$\sim 250 \mathrm{kDa}$ (Fig 1A, lanes 4, 6). This immunopositive staining was removed by digestion with keratanase (Fig. 1A, lanes 3, 5).

Monoclonal antibodies 1B5, 2B6, and 3B3 showed the predominant CS/DS PG to contain the 4-sulfated stub isomer present on a CS PG core protein (Fig. 1B, lane 4). 1B5 gave only weak staining, whereas the nitrocellulose was unstained with $3 \mathrm{~B} 3$, indicating trace and undetectable amounts of 0- and 6-sulfated CS, respectively (Fig. 1B, lanes 2, 7). A strong immunostaining band was observed at approximately $37 \mathrm{kDa}$ with 2B6 antibody after chondroitinase ABC digestion, representing neoepitope potentially generated from 4-sulfated CS and/or DS chains (Fig. 1B, lane 4). In contrast, chondroitinase AC II digestion produced a fainter band at 37 $\mathrm{kDa}$, plus a spread of immunoreactivity across the nitrocellulose at higher molecular weight. This probably represented partially digested CS/DS PGs with either both CS and DS or CS/DS copolymers substituted on their core proteins (Fig. 1B, lane 5).

Mouse corneal PG extracts isolated under reducing conditions and run on Tris-glycine gradient gels consistently revealed contamination at 25 and $50 \mathrm{kDa}$ by endogenous mouse IgG light and heavy chains, respectively (Fig. 1). This reflected a relatively high concentration of endogenous immunoglobulin, for a nonvascularized tissue such as the corneal stroma. The presence of IgG necessitated modifications to immunohistochemistry methodology, using anti-mouse secondary antibodies, as will be detailed later.

\section{Stromal Localization of Specific PGs by Immunohistochemistry}

Immunolabeling of mouse corneal PGs was successfully achieved using monoclonal antibodies and anti-mouse secondary antibodies when we used a one-step incubation of sections in a mixture of primary-secondary antibody complexes, supplemented with normal mouse serum. ${ }^{29}$ Both conventional two-step staining and incubation with complexes, or secondary antibody alone in the absence of normal serum, generated overwhelming levels of background fluorescence, presumably owing to binding of the secondary antibody to endogenous mouse corneal IgG (Fig. 2A). Control sections exposed to secondary antibody plus serum, but with primary antibody omitted, displayed low levels of background fluorescence (Fig. 2B).

In general, immunohistochemistry confirmed the results of Western blot analysis. Thus, antibody 5D4 against minimally pentasulfated disaccharides on KS consistently gave negative results (Fig. 2C, 2D), whereas relatively strong fluorescence was observed with antibody $1 \mathrm{~B} 4$, specific for lower-sulfated linear sulfation epitopes (Fig. 2F). This 1B4 signal was eradicated by keratanase pretreatment, thus verifying KS staining (Fig. 2G). A positive control for the 5D4 antibody was demonstrated by immunofluorescence staining of bovine articular cartilage using the same dilutions of primary and secondary antibody (Fig. 2E).

In line with the results from Western blot analysis, only low levels of staining were obtained with antibody 1B5, which recognized chondroitinase-generated 0 -sulfated CS stubs on CS/DS PGs, whereas results with 3B3, which recognizes the 6-sulfated stub isomer, were consistently negative (results not shown). However, a strong positive immunoreactivity was detected throughout the stroma to monoclonal antibody $2 \mathrm{~B} 6$, which recognized the 4-sulfated isomer stubs (Figs. 2I, 2J). As predicted, this immunostaining was dependent on chondroitinase digestion to expose the neoepitope for antibody $2 \mathrm{~B} 6$ (Figs. 2I, 2L). Levels of immunostaining with 2B6 antibody after chondroitinase AC II treatment were similar to those obtained after chondroitinase $\mathrm{ABC}$, indicating that $\mathrm{CS}$ was the predominant GAG present (Figs. 2J, 2M). Similar immunopositive staining patterns were observed with antibody $2 \mathrm{~B} 6$ in control sections of articular cartilage after chondroitinase $\mathrm{ABC}$ or AC II pretreatment (Figs. 2K, 2N).

\section{Unique Ultrastructure of Mouse Corneal PGs Visualized by Cationic Dye Staining}

Cupromeronic blue staining patterns revealed two populations of mouse corneal PGs (Fig. 3). A large number of fine filaments could be seen radiating from the collagen fibrils in the longitudinal section, where they sometimes exhibited regular associations with the D-periodic bands of the positively stained fibrils. In addition, much larger dye-positive structures with diameters up to $20 \mathrm{~nm}$ and maximum measurable length of approximately $600 \mathrm{~nm}$ were present. These conspicuous structures appeared variously as sinuously curved or linear elements, running at various angles with respect to the fibril axis within the lamellae and with threadlike extensions arising at intervals along them, sometimes making contact with adjacent collagen fibrils.

The cupromeronic-blue-positive structures responded differently to incubation with GAG-degrading enzymes (Fig. 

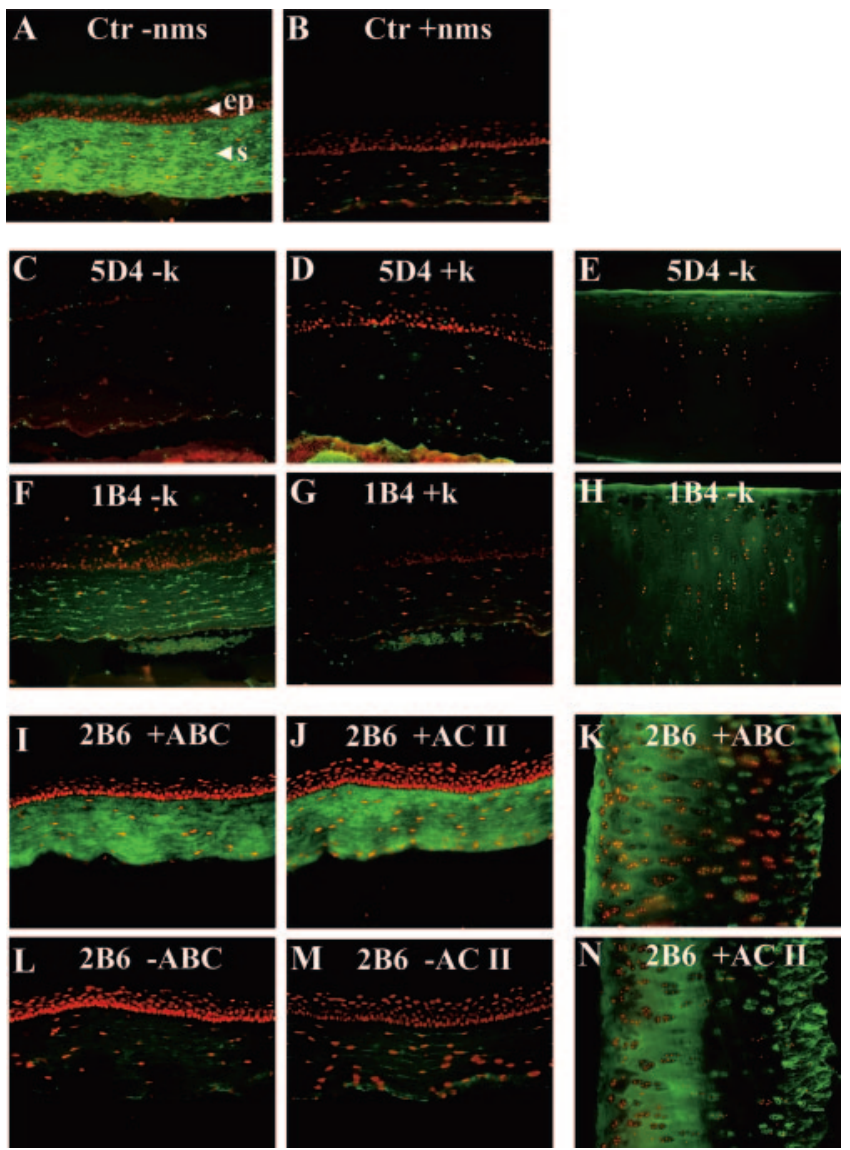

FIGURE 2. Immunofluorescence detection of PGs in mouse cornea with monoclonal antibodies against specific sulfated KS and CS/DSGAGs. Positive staining was shown by FITC (green), and Hoechst 33258 dye (red) counterstained the cell nuclei. (A) A high level of nonspecific fluorescence in mouse cornea in a control section, from which the primary antibody was omitted, was attributed to staining of endogenous mouse IgG by anti-mouse secondary antibody in the absence of normal mouse serum. ep, corneal epithelium; s, corneal stroma. (B) Background staining was eradicated in a control section when serum was included and antibody solutions were applied using the method described by Hierck et al. ${ }^{29}$ (C, D) Immunolocalization of mouse corneal KS PGs with antibody 5D4 was negative, indicating that no highly sulfated KS epitopes were detectable. In contrast, low sulfated KS was labeled by antibody $1 \mathrm{~B} 4$ (F). This staining was removed by keratanase pretreatment (G). Both 5D4 (E) and 1B4 (H) antibodies gave positive immunofluorescence in bovine articular cartilage. (I, J) Immunolocalization of chondroitinase-generated chondroitin 4-sulfate isomer stubs with antibody $2 \mathrm{~B} 6$ throughout the corneal stroma with no clear difference in signal intensity between sections pretreated with either chondroitinase $\mathrm{ABC}$ or chondroitinase AC II. (L, M) Staining was absent without enzyme predigestion to expose the neoepitope for the 2 B6 antibody. (K, N) As with mouse cornea, bovine articular cartilage showed positive staining with 2B6 after either enzyme pretreatment.

4). Chondroitinase $\mathrm{ABC}$ pretreatment removed the large filaments (Figs. 4G-I), although they were resistant to digestion by keratanase I (Figs. 4D-F). Numerous fine filaments were resistant to keratanase and were often located along the collagen fibrils. Chondroitinase ABC enzyme treatment left only poorly contrasted, dye-positive structures, which appeared smaller than those remaining after keratanase digestion and could sometimes be assigned to "a/c" collagen bands. No clear differences were evident between the anterior, mid, and posterior zones of the mouse corneal stroma.

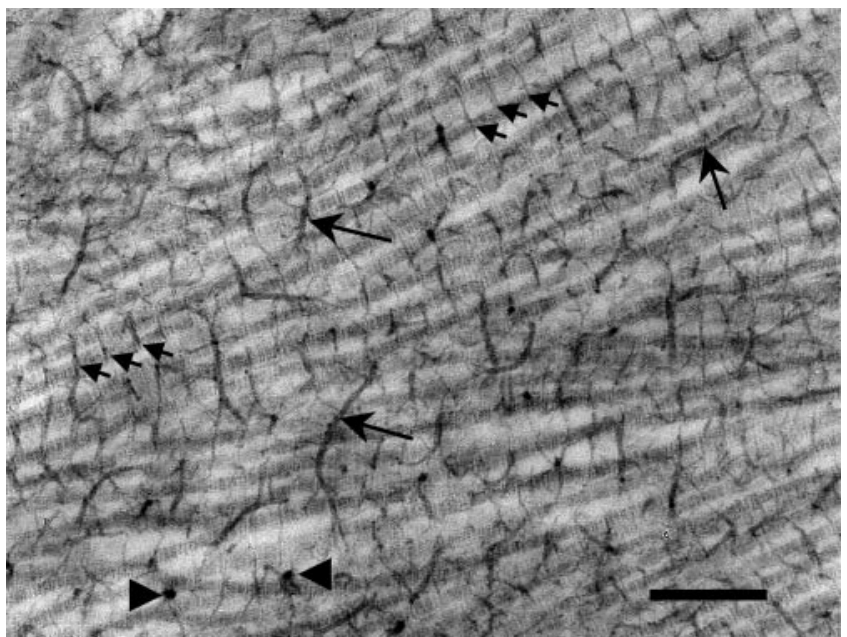

FIGURE 3. Ultrastructure of PGs in mouse cornea after contrast-enhanced fixation with cupromeronic blue. PGs appeared as electron dense filaments, sometimes regularly associated with the collagen fibrils (short arrows) seen in the longitudinal section, and as large, elongated structures which ran along and across the fibrils (long arrows). These large structures appeared circular in a transverse section, up to $20 \mathrm{~nm}$ in diameter, and with radiating extensions (arrowbeads). Bar, $250 \mathrm{~nm}$.

\section{DisCussion}

Investigations by electron microscopy have shown close and specific interactions between PGs and collagen fibrils in a range of connective tissues including tendon, ${ }^{30-32}$ bone, ${ }^{33}$ intervertebral disc, ${ }^{34}$ articular cartilage, ${ }^{35}$ sclera, ${ }^{36,37}$ and cornea. ${ }^{38-40}$ In the cornea, these regular and characteristic interactions support the hypothesis that PGs fulfill an essential role in the maintenance of matrix integrity and function with crucial importance for regulating collagen fibril diameter and tissue transparency. The introduction of gene targeted mutant mice with deficiencies in PGs currently offers great potential in furthering our understanding of the functions of these impor-
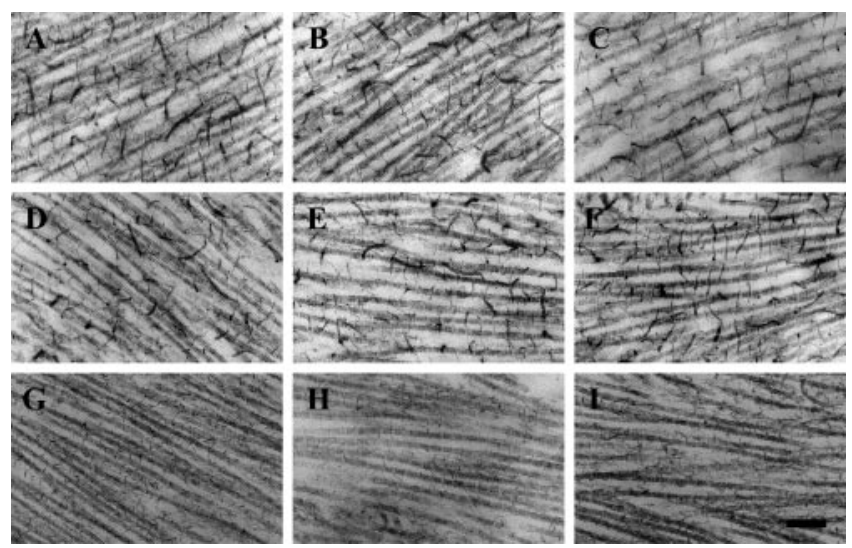

FIGURE 4. Effects of enzyme pretreatment on the structure of cupromeronic blue-contrasted PGs in anterior, mid, and posterior stroma of mouse cornea (left, middle, and right columns, respectively). A normal complement of PGs was present in cornea that was incubated in buffer without enzyme (A-C). Keratanase treatment (D-E) has little effect, apart from some slight reduction in collagen-associated filaments. Chondroitinase ABC (G-I) treatment removes large PG structures leaving variable amounts of collagen-associated filaments. Chondroitinase-resistant material (G-I) appeared as small, low-contrast filaments, presumed to represent KS PGs. Bar, $200 \mathrm{~nm}$. 
tant molecules in the cornea. ${ }^{41,42}$ Recent work has shown that a deficiency in lumican leads to abnormally large collagen fibrils in skin and cornea and to opacification of the cornea, ${ }^{16}$ whereas mice deficient in mimecan or keratocan also exhibit larger fibrils and disturbed fibril organization, but without loss of tissue transparency. ${ }^{19,21}$

Interpretation of the role of corneal PGs from studies of genetically altered mouse strains is crucially dependent on an appreciation of PG composition in the normal animal. Findings in earlier studies of mouse corneal PGs, in particular KS, are controversial. This PG was detectable in mouse corneal stroma by immunolocalization with the monoclonal antibody MZ15, ${ }^{43}$ yet electron microscopy and biochemical studies showed it to be absent, or at undetectable levels, compared to rat and rabbit cornea. ${ }^{44}$ A subsequent comparative study of corneas in several vertebrates found KS was indeed present in mouse cornea, but suggested that it lacked oversulfated disaccharide motifs. ${ }^{23}$ In this respect, mouse cornea appears to differ significantly from corneas in other mammalian species, where the KS PGs lumican and keratocan carry highly sulfated KS chains. Recently, Ying et al. ${ }^{45}$ found lumican core protein without KS chains to be abundant in neonatal mouse cornea by day 10 , with sulfated KS evident after the eyes opened, although the extent of sulfation was not assessed in this study. Keratan sulfate chains may also be absent from mimecan in mouse cornea, in contrast to bovine and human corneas. ${ }^{21}$

To clarify PG composition and structure in mouse cornea, we performed an investigation involving a range of antibodies sensitive to specific variations in sulfation patterns on KS and CS PGs. Immunoblot analysis of corneal PG extracts demonstrated a low level of reactivity with antibody $5 \mathrm{D} 4$, but relatively strong signal with antibodies $1 \mathrm{~B} 4$ and $4 \mathrm{D} 1$, thus confirming the absence of extended sequences of disulfated disaccharides on KS-GAGs in the mouse. These observations were corroborated by immunolocalization showing lower sulfated KS PG distributed evenly throughout the stromal thickness. Chondroitin 4-sulfate was the most readily detectable CS PG, with 0-sulfated CS also present, but no 6-sulfated CS PG identified. Previous studies on other connective tissues have shown a consistent increase in the ratio of 6:4 sulfated CS PG with age. ${ }^{46,47}$ No studies of CS 6:4 sulfate ratios with age have yet been performed on mouse cornea, but corneas examined in the present study were from young adults and the possibility that C-6-S may appear in the stroma of aged mice cannot be excluded. Differential digestion of mouse corneal C-4-S PG with chondroitinase $\mathrm{ABC}$ or AC II demonstrated an underlying heterogeneity of CS/DS-substituted PGs reflected in altered staining patterns on immunoblots with antibody $2 \mathrm{~B} 6$. According to one earlier report, mouse cornea may contain more than $60 \%$ of CS/DS PG as DS. ${ }^{23}$ Our results also indicate the presence of DS. They further indicate that most CS is 4-sulfated rather than 0- or 6-sulfated. We contend that CS chains in mouse cornea may exhibit structural diversity similar to that recently described in bovine cornea, ${ }^{48}$ and that core protein substituted with co-polymeric CS/DS chains, as well as with CS and DS chains individually, may coexist in the mouse stroma.

The ultrastructural organization of mouse corneal PGs, visualized with cupromeronic blue, is evidently more complex than that described previously in other mammalian corneas. Broadly, two types of dye-PG complexes were identifiable in the present study: fine collagen-associated filaments and unusually large, straplike structures. Dye-positive fine filamentous PGs are reminiscent of those previously described in other species. However, the most striking feature of corneas examined by electron microscopy was the presence of a population of large, intensely dye-positive complexes found draped along or wrapped around collagen fibrils in longitudinal section. We believe that these PGs represent structures unique to the mouse cornea and although their presence has been illustrated once previously in the literature, ${ }^{44}$ they have hitherto been overlooked as the attention of most researchers has been focused on the site-specific binding of the small collagenassociated PGs in this tissue. However, the large PG complexes constitute an abundant component of the stroma and they may therefore confer on the mouse cornea some important functional properties. Published studies of corneas from other rodent species ${ }^{44,49}$ have not revealed PG structures resembling these in mouse. To date, our attempts to characterize the large PGs with specific antibodies by immunoelectron microscopy have been unsuccessful. Nevertheless, we show in this study that they are resistant to digestion by keratanase enzyme, but are removed by chondroitinase $\mathrm{ABC}$ and thus most likely carry CS/DS-GAG chains. It remains to be determined whether increased sulfation of CS/DS PGs compensates for the undersulfation of KS PG in the mouse cornea. A maximum length of 600 $\mathrm{nm}$ was recorded for these structures, although the possibility cannot be excluded that these are merely portions, viewed in two dimensions, of longer structures running a continuous, but tortuous, course throughout the tissue. Although their electron-dense core component showed no regular association with stromal collagen fibrils, occasionally threadlike lateral extensions were present that appeared to make contact with the fibrils. They may therefore have a role in maintaining lamellar integrity.

In conclusion, there appear to be distinctive differences between mouse corneal PGs and those described in other animal species. The KS-GAGs on KS PGs seem to contain more $\mathrm{N}$-acetyl lactosamine disaccharides that are nonsulfated with no extended (pentasaccharide) domains containing disulfated disaccharide. Alternatively, the KS-GAGs are of shorter chain length than a pentasaccharide. Both of these possibilities would explain the absence of 5D4 antibody reactivity in mouse cornea. In addition, large chondroitinase ABC-sensitive PGs are present that, from their cationic dye staining structure, appear to have numerous CS/DS chains. We suggest that these unusual aspects of the mouse cornea be taken into account in the interpretation of data derived from studies of corneas in transgenic mouse strains.

\section{References}

1. Maurice DM. The structure and transparency of the corneal stroma. J Physiol. 1957;136:263-286.

2. Benedek GB. Theory of transparency of the eye. Appl Opt. 1971; $10: 459-473$

3. Freund DE, McCally RL, Farrell RA. Direct summation of fields for light scattering by fibrils with applications to normal corneas. Appl Opt. 1986;25:2739-2746.

4. Farrell RA. Corneal transparency. In: Albert DM, Jacobiec SA, eds. Principles and Practice of Opbthalmology. Philadelphia: WB Saunders; 1994.

5. Rada JA, Cornuet PK, Hassell JH. Regulation of corneal collagen fibrillogenesis in vitro by corneal keratan sulfate proteoglycan (lumican) and decorin core proteins. Exp Eye Res. 1993;56:635648.

6. Schonherr E, Hausser H, Beavan L, Kresse H. Decorin-type I collagen interaction. J Biol Chem. 1995;270:8877-8883.

7. Vogel KG, Paulsson M, Heinegard D. Specific inhibition of type I and type II collagen fibrillogenesis by the small proteoglycans of tendon. Biochem J. 1984;223:587-597.

8. Borcherding MS, Blacik LJ, Sittig RA, et al. Proteoglycans and collagen fibre organization in human corneoscleral tissue. Exp Eye Res. 1975;21:59-70.

9. Hassell JR, Cintron C, Kublin C, Newsome DA. Proteoglycan changes during restoration of transparency in corneal scars. $\mathrm{Arch}$ Biochem Biophys. 1983;222:362-369. 
10. Funderburgh JL, Cintron C, Covington HI, Conrad GW. Immunoanalysis of keratan sulfate proteoglycan from corneal scars. Invest Ophthalmol Vis Sci. 1988;29:1116-1124.

11. Li W, Vergnes J-P, Cornuet PK, Hassell JR. cDNA clone to chick corneal chondroitin dermatan sulfate proteoglycan reveals identity to decorin. Arch Biochem Biophys. 1992;296:190-197.

12. Funderburgh JL, Hevelone ND, Roth MR, et al. Decorin and biglycan of normal and pathologic human corneas. Invest Opbthalmol Vis Sci. 1998;39:1957-1964.

13. Blochberger TC, Vergnes J-P, Hempel J, Hassell JR. cDNA to chick lumican (corneal keratan sulfate proteoglycan) reveals homology to the small interstitial proteoglycan gene family and expression in muscle and intestine. J Biol Chem. 1992;267:347-352.

14. Corpuz LM, Funderburgh JL, Funderburgh ML, Bottomley GS, Prakash S, Conrad GW. Molecular cloning and tissue distribution of keratocan: bovine corneal keratan sulfate proteoglycan 37A. J Biol Chem. 1996;271:9759-9763.

15. Funderburgh JL, Corpuz LM, Roth MR, Funderburgh ML, Tasheva ES, Conrad GW. Mimecan, the $25-\mathrm{kDa}$ corneal keratan sulfate proteoglycan, is a product of the gene producing osteoglycin. J Biol Chem. 1997;272:28089-28095.

16. Chakravarti S, Magnuson T, Lass JH, Jepsen KJ, LaMantia C, Carroll H. Lumican regulates collagen fibril assembly: skin fragility and corneal opacity in the absence of lumican. J Cell Biol. 1998;141: 1277-1286

17. Chakravarti S, Petroll WM, Hassell JR, et al. Corneal opacity in lumican-null mice: defects in collagen fibril structure and packing in the posterior stroma. Invest Opbthalmol Vis Sci. 2000;41:33653373.

18. Quantock AJ, Meek KM, Chakravarti S. An x-ray diffraction investigation of corneal structure in lumican-deficient mice. Invest $O p b$ thalmol Vis Sci. 2001;42:1750-1756.

19. Liu C-Y, Birk DE, Hassell JR, Kane B, Kao W W-Y. Keratocandeficient mice display alterations in corneal structure.J Biol Chem. 2003;278:21672-21677.

20. Meek KM, Quantock AJ, Boote C, Liu CY, Kao WW-Y. An X-ray scattering investigation of corneal structure in keratocan-deficient mice. Matrix Biol. 2003;22:467-475.

21. Tasheva ES, Koester A, Paulsen AQ, et al. Mimecan/osteoglycindeficient mice have collagen fibril abnormalities. Mol Vis. 2002;8: 407-415.

22. Haigh M, Gibson S, Scott JE. Keratan sulphate and the ultrastructure of the cornea: a comparison of rabbit, rat and mouse. Biochem Soc Trans. 1987;15:711-712.

23. Scott JE, Bosworth TR. A comparative biochemical and ultrastructural study of proteoglycan-collagen interactions in corneal stroma. Biochem J. 1990;270:491-497.

24. Scott JE, Bosworth TR. The comparative chemical morphology of the mammalian cornea. Basic Appl Histochem. 1990;34:35-42.

25. Caterson B, Christner JE, Baker JR. Identification of a monoclonal antibody that specifically recognises corneal and skeletal keratan sulphate. J Biol Chem. 1983;258:8848-8854.

26. Caterson B, Christner JE, Baker JR, Couchman JR. Production and characterization of monoclonal antibodies directed against connective tissue proteoglycans. Fed Proc. 1985;44:386-393.

27. Mehmet H, Scudder P, Tang PW, Hounsell EF, Caterson B, Feizi T. The antigenic determinants recognized by three monoclonal antibodies to keratan sulphate involve hepta- or larger oligosaccharides of the poly(N-acetyllactosamine) series. Eur J Biochem. 1986; $157: 385-391$

28. Caterson B, Brooks K, Sattsangi S, Ratcliffe A, Hardingham T, Muir $\mathrm{H}$. Factors affecting the determination of keratan sulphate using monoclonal antibodies in immunoassay procedures. In: Greiling $\mathrm{H}$, Scott JE, eds. Keratan Sulphate: Chemistry, Biology and Chemical Patbology. London: The Biochemical Society; 1989:199-204.
29. Hierck BP, Iperen LV, Gittenberger-de Groot AC, Poelmann RE. Modified indirect immunodetection allows study of murine tissue with mouse monoclonal antibodies. J Histochem Cytochem. 1994; 42:1499-1502

30. Scott JE. Collagen-proteoglycan interactions. Localization of proteoglycans in tendon by electron microscopy. Biochem J. 1980; 187:887-891.

31. Scott JE, Orford CR, Hughes EW. Proteoglycan-collagen arrangements in developing rat tail tendon. Biochem J. 1981;195:573581 .

32. Scott JE, Orford CR. Dermatan sulphate-rich proteoglycan associates with rat tail tendon collagen at the d-band in the gap region. Biochem J. 1981;197:213-216.

33. Scott JE, Haigh M. Proteoglycan-type I collagen fibril interactions in bone and non-calcifying connective tissues. Biosci Rep. 1985;5: $71-81$.

34. Scott JE, Haigh M. Proteoglycan-collagen interactions in intervertebral disc: a chondroitin sulphate proteoglycan associates with collagen fibrils in rabbit annulus fibrosus at the d-e bands. Biosci Rep. 1986;6:879-888.

35. Orford CR, Gardner DL. Proteoglycan association with collagen d band in hyaline articular cartilage. Connect Tissue Res. 1984;12: 345-348.

36. Young RD. The ultrastructural organisation of proteoglycans and collagen in human and rabbit scleral matrix. J Cell Sci. 1985;74: 95-104.

37. Quantock AJ, Meek KM. Axial electron density of human scleral collagen: location of proteoglycans by x-ray diffraction. Biopbys J. 1988;54:159-164.

38. Scott JE, Haigh M. "Small"-proteoglycan:collagen interactions: keratan sulphate proteoglycan associates with rabbit corneal collagen fibrils at the "a" and "c" bands. Biosci Rep. 1985;5:765-774.

39. Meek KM, Elliott GF, Nave C. A synchrotron x-ray diffraction study of bovine cornea stained with cupromeronic blue. Collagen Relat Res. 1986;6:203-218.

40. Quantock AJ, Meek KM. Proteoglycan distribution in the corneas of individuals with bullous keratopathy. Biochem Soc Trans. 1990; 18:958.

41. Chakravarti S. Functions of lumican and fibromodulin: lessons from knockout mice. Glycoconjugate J. 2003;19:287-293.

42. Kao WW-Y, Liu C-Y. Roles of lumican and keratocan on corneal transparency. Glycoconjugate J. 2003;19:275-285.

43. Venn G, Mason RM. Absence of keratan sulphate from skeletal tissues of mouse and rat. Biochem J. 1985;228:443-450.

44. Scott JE, Haigh M. Keratan sulphate and the ultrastructure of cornea and cartilage: a "stand-in" for chondroitin sulphate in conditions of oxygen lack? J Anat. 1988;158:95-108.

45. Ying S, Shiraishi A, Kao CW-C, et al. Characterization and expression of the mouse lumican gene. J Biol Chem. 1997;272:3030630313.

46. Bayliss MT, Osborne D, Woodhouse S, Davidson C. Sulfation of chondroitin sulphate in human articular cartilage: the effect of age, topographical position and zone of cartilage on tissue composition. J Biol Chem. 1999;274:15892-15900.

47. Lauder RM, Huckerby TN, Brown GM, Bayliss MT, Niedusynski IA. Age-related changes in the sulphation of the chondroitin sulphate linkage region from human articular cartilage aggrecan. Biochem J. 2001;358:523-528.

48. Achur RN, Muthusamy A, Madhunapantula SV, Bhavanandan VP, Seudieu C, Channe Gowda D. Chondroitin sulphate proteoglycans of bovine cornea: structural characterization and assessment for the adherence of Plasmodium falciparum-infected erythrocytes. Biochim Biophys Acta. 2004;1701:109-119.

49. Velasco A, Hidalgo J. Ultrastructural demonstration of proteoglycans in adult rat cornea. Tissue Cell. 1988;20:567-575. 